\title{
An Improved Physical ZKP for Nonogram
}

\author{
Suthee Ruangwises*1 \\ ${ }^{1}$ Department of Mathematical and Computing Science, Tokyo Institute of \\ Technology, Tokyo, Japan
}

\begin{abstract}
Nonogram is a logic puzzle consisting of a rectangular grid with an objective to color every cell black or white such that the lengths of blocks of consecutive black cells in each row and column are equal to the given numbers. In 2010, Chien and Hon developed the first physical zero-knowledge proof for Nonogram, which allows a prover to physically show that he/she knows a solution of the puzzle without revealing it. However, their protocol requires special tools such as scratch-off cards and a machine to seal the cards, which are difficult to find in everyday life. Their protocol also has a nonzero soundness error. In this paper, we propose a more practical physical zero-knowledge proof for Nonogram that uses only a deck of regular paper cards and also has perfect soundness.

Keywords: zero-knowledge proof, card-based cryptography, Nonogram, puzzle
\end{abstract}

\section{Introduction}

Nonogram, also known as Picross or Pic-a-Pix, is one of the most popular logic puzzles alongside Sudoku, Kakuro, and other puzzles. A large number of Nonogram mobile apps have been developed [7].

A Nonogram puzzle consists of a rectangular grid of size $m \times n$. The objective of this puzzle is to color every cell black or white according to the following constraints. In each row and each column, there is a sequence of numbers, say $\left(x_{1}, x_{2}, \ldots, x_{k}\right)$, assigned to it; this means the corresponding row (resp. column) must contain exactly $k$ blocks of consecutive black cells with lengths $x_{1}, x_{2}, \ldots, x_{k}$ in this order from left to right (resp. from top to bottom), with at least one white cell separating consecutive blocks. For instance, in Fig. 1. the sequence $(3,2)$ on the topmost row means that row must contain a block of three consecutive black cells, and another block of two consecutive black cells to the right of it, separated by at least one white cell.

Suppose that Percy created a difficult Nonogram puzzle and challenged his friend Violetta to solve it. After a while, Violetta could not solve his puzzle and began to doubt whether the puzzle has a solution. In order to to convince her that his puzzle actually has a solution without revealing it (which would make the challenge pointless), Percy needs a zero-knowledge proof (ZKP).

*ruangwises@gmail.com 

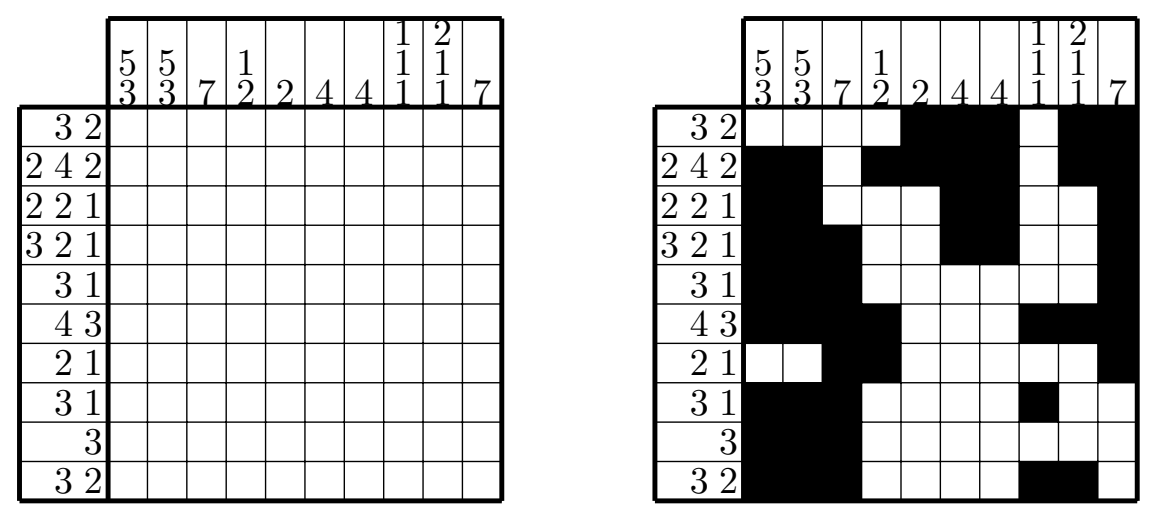

Figure 1: An example of a Nonogram puzzle (left) and its solution (right)

\subsection{Zero-Knowledge Proof}

A prover $P$ and a verifier $V$ are given a computational problem $x$, but only $P$ knows a solution $w$ of $x$. A ZKP is an interactive proof introduced by Goldwasser et al. [6], which allows $P$ to convince $V$ that he/she knows $w$ without revealing any information about it. A ZKP must satisfy the following properties.

1. Completeness: If $P$ knows $w$, then $P$ can convince $V$ with high probability. (Here we consider the perfect completeness property where the probability is one.)

2. Soundness: If $P$ does not know $w$, then $P$ cannot convince $V$, except with a small probability called soundness error. (Here we consider the perfect soundness property where the soundness error is zero.)

3. Zero-knowledge: $V$ learns nothing about $w$. Formally, there exists a probabilistic polynomial time algorithm $S$ (called a simulator) that does not know $w$ such that the outputs of $S$ and the outputs of the real protocol follow the same probability distribution.

Goldreich et al. 5 showed that a computational ZKP exists for every NP problem. As Nonogram is NP-complete [25, one can construct a computational ZKP for it via a reduction to another problem. Such construction, however, is not intuitive and looks unconvincing. Hence, many recent results instead focused on constructing physical ZKPs using portable objects such as a deck of cards and envelopes. These protocols have benefits that they do not require computers and allow external observers to check that the prover truthfully executes the protocol (which is often a challenging task for digital protocols). These intuitive protocols can also be used to teach the concept of ZKP to non-experts.

\subsection{Related Work}

Development of physical ZKPs for logic puzzles began in 2009 when Gradwohl et al. 8 proposed six ZKP protocols for Sudoku. Each of these protocols, however, either requires special tools or has a nonzero soundness error. Later, Sasaki et al. [22] improved the protocols for Sudoku to achieve perfect soundness without using special tools. Besides 
Sudoku, protocols for other puzzles have also been developed, including Akari [1], Takuzu [1, 12, Kakuro [1, 13, KenKen 11, Makaro [2], Norinori [4], Slitherlink [11, Juosan [12, Numberlink [19], Suguru [17, Ripple Effect [20], Nurikabe [16], and Hitori [16].

In 2010, Chien and Hon [3] proposed the first physical ZKP for Nonogram. However, their protocol requires scratch-off cards and a machine for the prover to seal the cards. These special tools cannot be easily found in everyday life. Moreover, their protocol has a nonzero soundness error. In fact, the soundness error is as high as $6 / 7$, which means practically, the protocol has to be repeated for many times until the soundness error becomes reasonably low.

\subsection{Our Contribution}

In this paper, we propose a more practical physical ZKP for Nonogram using only a portable deck of regular paper cards, which are easy to find in everyday life and can be reused. Our protocol also has perfect completeness and perfect soundness. In an $m \times n$ Nonogram puzzle with a total of $w$ white cells, our protocol uses $2 m n+2 \max (m, n)+6$ cards and $m n+2 m+2 n+2 w$ shuffles.

\section{Preliminaries}

\subsection{Cards}

We use four types of cards in our protocol: $\Omega, \varnothing, \emptyset$, and $\emptyset$. The front sides of cards in the same type are identical. The back sides of all cards are identical and are denoted by ?

\subsection{Random Cut}

Given a sequence of $k$ cards, a random cut rearranges the cards by a random cyclic shift, i.e. shifts the cards cyclically to the right by $r$ cards for a uniformly random $r \in\{0,1, \ldots, k-1\}$ unknown to all parties.

The random cut can be performed in real world by taking turns to apply several Hindu cuts to the sequence of cards [24].

\subsection{Pile-Shifting Shuffle}

Given an $\ell \times k$ matrix of cards, a pile-shifting shuffle rearranges the columns of the matrix by a random cyclic shift, i.e. shifts the columns cyclically to the right by $r$ columns for a uniformly random $r \in\{0,1, \ldots, k-1\}$ unknown to all parties.

The pile-shifting shuffle was developed by Shinagawa et al. [23]. It can be performed in real world by putting the cards in each column into an envelope and applying the random cut to the sequence of envelopes.

\subsection{Copy Protocol}

Given a sequence of two face-down cards of either 8 or 9 , a copy protocol creates an additional copy of the original sequence without revealing it. This protocol was developed by Mizuki and Sone [15]. 


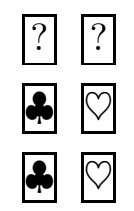

Figure 2: A $3 \times 2$ matrix constructed in Step 1 of the copy protocol

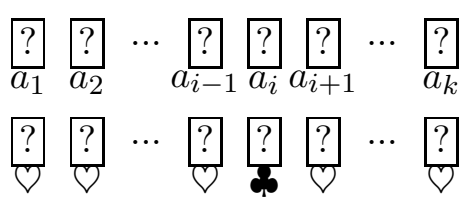

Figure 3: A $2 \times k$ matrix constructed in Step 1 of the chosen cut protocol

1. Construct a $3 \times 2$ matrix of cards by placing an original sequence in the first row, and a sequence $\$$ in each of the second and third rows (see Fig. 22).

2. Turn over all face-up cards. Apply the pile-shifting shuffle to the matrix.

3. Turn over the two cards in the first row. If the revealed sequence is 8 , do nothing; if the sequence is 8 , swap the two columns of the matrix.

4. The sequences in the second and third rows are the two copies of the original sequence as desired.

Note that this protocol also verifies that the original sequence is either $\theta$ or $P$. (and not 9 or $P[$ ).

\subsection{Chosen Cut Protocol}

Given a sequence of $k$ face-down cards $A=\left(a_{1}, a_{2}, \ldots, a_{k}\right)$, a chosen cut protocol allows the prover $P$ to select a card $a_{i}$ he/she wants without revealing $i$ to the verifier $V$. This protocol was developed by Koch and Walzer [9].

1. Construct the following $2 \times k$ matrix (see Fig. 3).

(a) In the first row, $P$ publicly places the sequence $A$.

(b) In the second row, $P$ secretly places a face-down $Q$ at every position except at the $i$-th column where $P$ places a face-down instead.

2. Apply the pile-shifting shuffle to the matrix.

3. Turn over all cards in the second row. The card above the only will be the card $a_{i}$ as desired. 


\section{Main Protocol}

First, $P$ secretly places a face-down sequence 90 on each black cell, and a face-down sequence $P$ on each white cell in the Nonogram grid according to his/her solution. Then, $P$ publicly applies the copy protocol to create an additional copy of the sequence on every cell. Each of the two copies will be used to verify the row and the column that cell is located on. Note that the copy protocol also verifies that each sequence is in a correct format (either

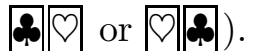

From now on, we will show the verification of a row $R$ with $n$ cells and with a sequence $\left(x_{1}, x_{2}, \ldots, x_{k}\right)$ assigned to it. (The verification of a column works analogously in the direction from top to bottom.)

$P$ takes only the left card from the sequence on each cell in $R$ (which is a for a black cell and is a $Q$ for a white cell) to form a sequence of cards $S=\left(a_{1}, a_{2}, \ldots, a_{n}\right)$, where each card corresponds to each cell in $R$ in this order from left to right. As $R$ may start and end with a white or black cell, $P$ publicly appends two $Q \mathrm{~s} a_{0}$ and $a_{n+1}$ at the beginning and the end of $S$, respectively, to ensure that the sequence starts and ends with a $Q$. Finally, $P$ publicly appends a marker card $\vartheta$, called $a_{n+2}$, at the end of $S$ to mark the end of the sequence. See Fig. 4 for an example.

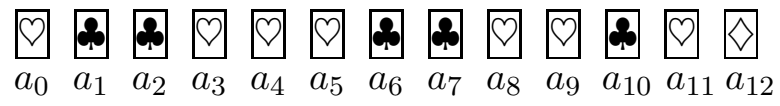

Figure 4: A sequence $S$ representing the third row of the solution in Fig 1

The verification is divided into the following three phases.

\subsection{Phase 1: Counting Black Cells}

Currently, there are $k$ blocks of consecutive $\mathrm{s}$ in $S$. In this phase, $P$ will reveal the length of each block as well as replacing every with a $\$$.

$P$ performs the following steps for $k$ rounds. In the $i$-th round:

1. Apply the chosen cut protocol to $S$ to select a card corresponding to the leftmost cell of the $i$-th leftmost block of black cells in $R$ (the block with length $x_{i}$ ). Let $a_{j}$ denote the selected card.

2. Turn over cards $a_{j}, a_{j+1}, a_{j+2}, \ldots, a_{j+x_{i}-1}$ to reveal that they are all $\mathrm{g}_{\mathrm{s}}$ (otherwise $V$ rejects), where the indices are taken modulo $n+3$.

3. Turn over cards $a_{j-1}$ and $a_{j+x_{i}}$ to reveal that they are both $Q \mathrm{~s}$ (otherwise $V$ rejects), where the indices are taken modulo $n+3$.

4. Replace every face-up with a $\$$. Turn over all face-up cards.

After $k$ rounds, $V$ is now convinced that there are $k$ different blocks of black cells with lengths $x_{1}, x_{2}, \ldots, x_{k}$, but still does not know about the order of these blocks, or whether there are any additional blocks of black cells besides these $k$ blocks. Note that all $\mathrm{s}$ s in $S$ is now replaced by $\mathbf{s}$. See Fig. 5 for an example. 
Figure 5: The sequence $S$ from Fig. 4 at the end of Phase 1

\subsection{Phase 2: Removing White Cells}

Currently, there are $k+1$ blocks of consecutive $\nabla \mathrm{s}$ in $S$ (including a block at the beginning containing $a_{0}$ and a block at the end containing $a_{n+1}$ ). In this phase, $P$ will remove some $\nabla$ s from $S$ such that there will be exactly one remaining $\square$ in each block.

Let $X=x_{1}+x_{2}+\ldots+x_{k}$. There are $n-X$ white cells in $R$, so there are currently $n-X+2$ Ds in $S$. $P$ performs the following steps for $(n-X+2)-(k+1)=n-X-k+1$ rounds.

1. Apply the chosen cut protocol to $S$ to select any $Q$ such that there are currently at least two remaining $\mathrm{P}$ s in a block it belongs to.

2. Turn over the selected card to reveal that it is a $P$ (otherwise $V$ rejects).

3. Remove that card from $S$.

Now, each pair of consecutive blocks of $\$$ s are separated by exactly one $Q$, and there is also a $Q$ at the beginning and at the end of $S$. See Fig. 6 for an example.

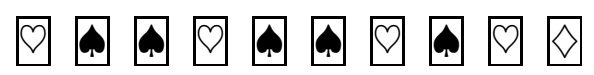

Figure 6: The sequence $S$ from Fig. 4 at the end of Phase 2

\subsection{Phase 3: Verifying Order of Blocks}

$P$ applies the random cut to $S$, turns over all cards, and shifts the sequence cyclically such that the rightmost card is a $\nabla$. $V$ verifies that the remaining cards in $S$ are: a $Q$, a block

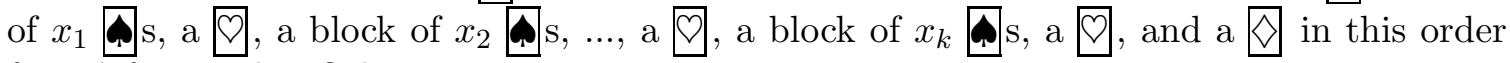
from left to right. Otherwise, $V$ rejects.

$P$ performs the verification for every row and column of the grid. If all rows and columns pass the verification, then $V$ accepts.

\subsection{Optimization}

In fact, at the beginning we do not need to copy a sequence on each cell since we only use one card per cell during the verification. Instead, we perform the copy protocol without putting cards in the third row of the matrix in Step 1. This modified protocol verifies that the original sequence is either 9 or $Q 9$, and then returns the original sequence in the second row. This modified protocol was developed by Mizuki and Shizuya [14].

After verifying that each sequence is in a correct format, we use the left card to verify a row, and the right card to verify a column that cell is located on. When verifying a column, the corresponding card is a $P$ for a black cell and a for a white cell, so we treat $\rightarrow$ and 0 exactly the opposite way as described in Section 3 , 
After the optimization, our protocol uses $m n+1$ s, $m n+\max (m, n)+4$ s, $\max (m, n)$ $\$ \mathrm{~s}$, and one $\nabla$, resulting in a total of $2 m n+2 \max (m, n)+6$ cards. It uses $m n+2 m+2 n+2 w$ shuffles, where $w$ is the total number of white cells in the grid.

\section{Proof of Security}

We will prove the perfect completeness, perfect soundness, and zero-knowledge properties of our protocol.

Lemma 1 (Perfect Completeness). If $P$ knows a solution of the Nonogram puzzle, then $V$ always accepts.

Proof. Suppose $P$ knows a solution of the puzzle. Consider the verification of any row $R$.

In Phase 1 , in each $i$-th round $P$ selects a card $a_{j}$ corresponding to the leftmost cell of the $i$-th leftmost block of black cells in $R$. Since that block has length $x_{i}$ and has never been selected before, all of the cards $a_{j}, a_{j+1}, a_{j+2}, \ldots, a_{j+x_{i}-1}$ must be $\mathrm{s}$. Also, since there is at least one white cell between two consecutive blocks of black cells (and at least one $P$ to the left of the leftmost block of $\mathrm{s}$ and to the right of the rightmost block of $\mathrm{s}$ ), both of the cards $a_{j-1}$ and $a_{j+x_{i}}$ must be $\emptyset$ s. Hence, the verification will pass Phase 1 .

At the start of Phase 2, there are a total of $n-X+2 \square \mathrm{s}$ in $k+1$ blocks of $\square \mathrm{s}$, so $P$ can remove a $P$ in each round for $(n-X+2)-(k+1)=n-X-k+1$ rounds such that there will be exactly one remaining $Q$ in each block. Hence, the verification will pass Phase 2.

In Phase 3, there is exactly one $Q$ between two consecutive blocks of $\$ \mathrm{~s}$ (and a $Q$ at the beginning and the end of $S$ ). Moreover, the blocks of $\$$ s are arranged in exactly the same order as the blocks of black cells, so the lengths of these blocks are $x_{1}, x_{2}, \ldots, x_{k}$ in this order from left to right. Hence, the verification will pass Phase 3.

Since this is true for every row (and analogously for every column), $V$ will always accept.

Lemma 2 (Perfect Soundness). If $P$ does not know a solution of the Nonogram puzzle, then $V$ always rejects.

Proof. We will prove the contrapositive of this statement. Suppose $V$ accepts, meaning that the verification must pass for every row and column. We will prove that $P$ must know a solution.

Consider the verification of any row $R$. In Phase 1 , the steps in each $i$-th round ensure that there exists a block of exactly $x_{i}$ consecutive black cells in $R$. Also, since all $\rightarrow \mathrm{s}$ selected in previous rounds have been replaced with $\$ \mathrm{~s}$, this block must be different from the blocks revealed in previous rounds. Therefore, there must be at least $k$ different blocks of black cells with lengths $x_{1}, x_{2}, \ldots, x_{k}$ (in some order) in $R$.

Moreover, in Phase 2 only $P$ s are removed from $S$, and when all cards in $S$ are turned face-up in Phase 3 there is no 0 remaining. This implies $R$ has no other black cells besides the ones in these $k$ blocks.

During Phase 3 , the lengths of the blocks of $\$ \mathrm{~s}$ are $x_{1}, x_{2}, \ldots, x_{k}$ in this order from left to right. As the blocks of $\$ \mathrm{~s}$ are arranged in exactly the same order as the blocks of black 
cells, the lengths of the blocks of black cells must also be $x_{1}, x_{2}, \ldots, x_{k}$ in this order from left to right.

Since this is true for every row (and analogously for every column), $P$ must know a solution of the puzzle.

Lemma 3 (Zero-Knowledge). During the verification, $V$ learns nothing about $P$ 's solution.

Proof. To prove the zero-knowledge property, it is sufficient to show that all distributions of cards that are turned face-up can be simulated by a simulator $S$ without knowing $P$ 's solution.

- In Step 3 of the copy protocol, the revealed sequence has an equal probability to be Q $P$ or $P$, so this step can be simulated by $S$.

- In Step 3 of the chosen cut protocol, the has an equal probability to be at any of the $k$ positions, so this step can be simulated by $S$.

- In the main protocol, there is only one specific pattern of the cards that are turned face-up in every phase, so the whole protocol can be simulated by $S$.

\section{Future Work}

We developed a physical ZKP for Nonogram using $2 m n+2 \max (m, n)+6$ cards and $m n+$ $2 m+2 n+2 w$ shuffles. A challenging future work is to develop a ZKP for this puzzle that can be performed using a standard deck (a deck containing all different cards), an open problem posed in [10. Other possible future work includes developing ZKPs for other popular logic puzzles or improving the already existing ones, as well as exploring methods to physically verify other numerical functions.

\section{References}

[1] X. Bultel, J. Dreier, J.-G. Dumas and P. Lafourcade. Physical Zero-Knowledge Proofs for Akari, Takuzu, Kakuro and KenKen. In Proceedings of the 8th International Conference on Fun with Algorithms (FUN), pp. 8:1-8:20 (2016).

[2] X. Bultel, J. Dreier, J.-G. Dumas, P. Lafourcade, D. Miyahara, T. Mizuki, A. Nagao, T. Sasaki, K. Shinagawa and H. Sone. Physical Zero-Knowledge Proof for Makaro. In Proceedings of the 20th International Symposium on Stabilization, Safety, and Security of Distributed Systems (SSS), pp. 111-125 (2018).

[3] Y.-F. Chien and W.-K. Hon. Cryptographic and Physical Zero-Knowledge Proof: From Sudoku to Nonogram. In Proceedings of the 5th International Conference on Fun with Algorithms (FUN), pp. 102-112 (2010).

[4] J.-G. Dumas, P. Lafourcade, D. Miyahara, T. Mizuki, T. Sasaki and H. Sone. Interactive Physical Zero-Knowledge Proof for Norinori. In Proceedings of the 25th International Computing and Combinatorics Conference (COCOON), pp. 166-177 (2019). 
[5] O. Goldreich, S. Micali and A. Wigderson. Proofs that yield nothing but their validity and a methodology of cryptographic protocol design. Journal of the ACM, 38(3): 691729 (1991).

[6] S. Goldwasser, S. Micali and C. Rackoff. The knowledge complexity of interactive proof systems. SIAM Journal on Computing, 18(1): 186-208 (1989).

[7] Google Play: Nonogram. https://play.google.com/store/search?q=Nonogram

[8] R. Gradwohl, M. Naor, B. Pinkas and G.N. Rothblum. Cryptographic and Physical Zero-Knowledge Proof Systems for Solutions of Sudoku Puzzles. Theory of Computing Systems, 44(2): 245-268 (2009).

[9] A. Koch and S. Walzer. Foundations for Actively Secure Card-Based Cryptography. In Proceedings of the 10th International Conference on Fun with Algorithms (FUN), pp. 17:1-17:23 (2020).

[10] H. Koyama, D. Miyahara, T. Mizuki and H. Sone. A Secure Three-Input AND Protocol with a Standard Deck of Minimal Cards. In Proceedings of the 16th International Computer Science Symposium in Russia (CSR), pp. 242-256 (2021).

[11] P. Lafourcade, D. Miyahara, T. Mizuki, L. Robert, T. Sasaki and H. Sone. How to construct physical zero-knowledge proofs for puzzles with a "single loop" condition. Theoretical Computer Science, 888: 41-55 (2021).

[12] D. Miyahara, L. Robert, P. Lafourcade, S. Takeshige, T. Mizuki, K. Shinagawa, A. Nagao and H. Sone. Card-Based ZKP Protocols for Takuzu and Juosan. In Proceedings of the 10th International Conference on Fun with Algorithms (FUN), pp. 20:1-20:21 (2020).

[13] D. Miyahara, T. Sasaki, T. Mizuki and H. Sone. Card-Based Physical Zero-Knowledge Proof for Kakuro. IEICE Transactions on Fundamentals of Electronics, Communications and Computer Sciences, E102.A(9): 1072-1078 (2019).

[14] T. Mizuki and H. Shizuya. Practical Card-Based Cryptography. In Proceedings of the 7th International Conference on Fun with Algorithms (FUN), pp. 313-324 (2014).

[15] T. Mizuki and H. Sone. Six-Card Secure AND and Four-Card Secure XOR. In Proceedings of the 3rd International Frontiers of Algorithmics Workshop (FAW), pp. 358-369 (2009).

[16] L. Robert, D. Miyahara, P. Lafourcade and T. Mizuki. Interactive Physical ZKP for Connectivity: Applications to Nurikabe and Hitori. In Proceedings of the 17th Conference on Computability in Europe (CiE), pp. 373-384 (2021).

[17] L. Robert, D. Miyahara, P. Lafourcade and T. Mizuki. Physical Zero-Knowledge Proof for Suguru Puzzle. In Proceedings of the 22nd International Symposium on Stabilization, Safety, and Security of Distributed Systems (SSS), pp. 235-247 (2020).

[18] S. Ruangwises. Two Standard Decks of Playing Cards Are Sufficient for a ZKP for Sudoku. In Proceedings of the 27th International Computing and Combinatorics Conference (COCOON), pp. 631-642 (2021). 
[19] S. Ruangwises and T. Itoh. Physical Zero-Knowledge Proof for Numberlink Puzzle and $k$ Vertex-Disjoint Paths Problem. New Generation Computing, 39(1): 3-17 (2021).

[20] S. Ruangwises and T. Itoh. Physical Zero-Knowledge Proof for Ripple Effect. In Proceedings of the 15th International Conference and Workshops on Algorithms and Computation (WALCOM), pp. 296-307 (2021).

[21] S. Ruangwises and T. Itoh. Physical ZKP for Connected Spanning Subgraph: Applications to Bridges Puzzle and Other Problems. In Proceedings of the 19th International Conference on Unconventional Computation and Natural Computation (UCNC), pp. 149-163 (2021).

[22] T. Sasaki, D. Miyahara, T. Mizuki and H. Sone. Efficient card-based zero-knowledge proof for Sudoku. Theoretical Computer Science, 839: 135-142 (2020).

[23] K. Shinagawa, T. Mizuki, J.C.N. Schuldt, K. Nuida, N. Kanayama, T. Nishide, G. Hanaoka and E. Okamoto. Card-Based Protocols Using Regular Polygon Cards. IEICE Transactions on Fundamentals of Electronics, Communications and Computer Sciences, E100.A(9): 1900-1909 (2017).

[24] I. Ueda, D. Miyahara, A. Nishimura, Y. Hayashi, T. Mizuki and H. Sone. Secure implementations of a random bisection cut. International Journal of Information Security, 19(4): 445-452 (2020).

[25] N. Ueda and T. Nagao. NP-completeness Results for NONOGRAM via Parsimonious Reductions. Technical Report TR96-0008, Department of Computer Science, Tokyo Institute of Technology (1996). 